\title{
Introducción al conocimiento de los hongos comestibles en cinco localidades de la Huasteca, Hidalguense, México
}

\author{
Introduction to the knowledge of edible fungi in five localities of the Huasteca, Hidalguense, \\ Mexico
}

\section{Juan Cipriano-Anastasio ${ }^{a}$,Alejandra López-Mancilla ${ }^{b}$, Rosalba Galván-Gutiérrez ${ }^{b}$, Germán Hernández-Alvarado ${ }^{c}$,Ericka Hernández-Hernández $z^{c}$ Ricardo Marcos-Méndez ${ }^{c}$.}

\begin{abstract}
:
The great biological diversity that Mexico has is an essential factor for wealth as it corresponds to the fungi kingdom, the Sierra and Huasteca Hidalguense has very diverse ecosystems that allow a greater diversity of fungi. The main objective of the present study is to know the use that the inhabitants give to the fungi in five localities of the Huasteca, Hidalguense. Visits were made during the month of September 2017, making a total of 52 open interviews in total to adults over 40 years. The only use that was found is food, presenting seven different ways of stewing (Tlapanile, guisado, caldo, tamales, quesadillas, adobo y hervidos). The most used fungus is called yellow or Chiquinte. The villagers refer to mushrooms a pleasant flavor similar to fish. According to the information obtained, a total of three genera and two morphospecies (Cantharellus, Pleurotus, Auricularia, Ustilago maydis and Agaricus bisporus). Nine local names were obtained for the fungi. Some names refer to the same species. As for its season, they are obtained during the rainy season and on different substrates. The differentiation of edible and non-edible fungi was based on the colors mentioned by the villagers. Edible: purple, white and yellow, inedible: red and gray. It is important to mention that the inhabitants of the five localities still conserve the knowledge on the edible wild mushrooms.
\end{abstract}

\section{Keywords:}

Mushroom, Stew, Season, Common Names, Huasteca Hidalguense

\section{Resumen:}

La gran diversidad biológica con la que cuenta México es un factor esencial para la riqueza en cuanto corresponde al reino fungi, la Sierra y Huasteca Hidalguense cuenta con ecosistemas muy diversos que permiten una mayor diversidad de hongos. El objetivo principal del presente estudio es conocer el uso que los habitantes le dan a los hongos en cinco localidades de la Huasteca, Hidalguense. Se realizaron visitas durante el mes de septiembre del 2017, efectuando un total de 52 entrevistas abiertas en total a personas adultas mayores de 40 años. El único uso que se encontró es alimenticio, presentándose siete diferentes maneras de guisado (Tlapanile, guisado, caldo, tamales, quesadillas, adobo y hervidos). El hongo más utilizado es el llamado amarillo o Chiquinte. Los pobladores hacen referencia a los hongos un agradable sabor similar al pescado. De acuerdo con la información recabada, se obtuvieron un total de tres géneros y dos morfoespecies (Cantharellus, Pleurotus, Auricularia, Ustilago maydis y Agaricus bisporus). Se obtuvieron nueve nombres locales para los hongos. Algunos nombres hacen referencia a la misma especie. En cuanto a su temporada, son obtenidos en época de lluvias y en diferentes sustratos. La diferenciación de los hongos comestibles y no comestibles fue con base a los colores mencionados por los pobladores. Comestibles: morado, blanco y amarillo, no comestibles: rojo y gris. Es importante mencionar que los pobladores de las cinco localidades aún conservan el conocimiento sobre los hongos silvestres comestibles.

\section{Palabras Clave:}

Hongo, Guisado, Temporada, Nombres Comunes, Huasteca Hidalguense

\section{Introducción}

El reino Fungi representa uno de los más grandes componentes de la biodiversidad con actividades ecológicas cruciales en todos los ecosistemas como la sobrevivencia de otros organismos y con una gran variabilidad en morfología y ciclos de vida [1]. Los hongos son quizá el grupo biológico al que se le presta menos atención cuando se habla de las relaciones entre los pueblos y los recursos naturales de su entorno. Sin

\footnotetext{
${ }^{a}$ Autor de correspondencia, Instituto Tecnológico de Huejutla, Email: bio.jca@ gmail.com

b Instituto Tecnológico de Huejutla, Email: bioalm@ @otmail.com, rosalbagalvang@hotmail.com Instituto Tecnológico de Huejutla, Email: ghaalvarado94@hotmail.com, minionsmartinez981@gmail.com,
} 
embargo, otros grupos humanos los consideran de mucha utilidad. Se utilizan como alimento, medicina, ornamento, para venderlos en los mercados o intercambiarlos por algún otro bien [2]. Los hongos comestibles silvestres forman parte de la diversidad biológica, ecológica y cultural de México y han contribuido en la conformación del conocimiento tradicional, el cual data desde la época prehispánica, a su vez se calcula que son más de 200 especies comestibles para nuestro país y que esto se debe en el uso múltiple de los recursos naturales [3], [4].

En México, el consumo de los hongos comestibles silvestres es una actividad relativamente nueva que en algunos casos generan beneficios monetarios importantes para los recolectores [5], [6], [7]. El estado de Hidalgo ocupa el tercer lugar en diversidad de especies comestibles [8], [9]. A su vez se han realizado estudios etnomicológicos con especies como Pleurotus albidus (Berk.) [10] y Calostoma cinnabarina, Cantharellus cibarius, $C$. odoratus [11] en donde se documenta su importancia como un recurso consumible de los grupos humanos de la zona. Las personas que consumen hongos comestibles lo hacen por su sabor, aroma o textura, sin darse cuenta a veces de las propiedades medicinales y nutricionales que comprenden; por ejemplo, tienen un bajo contenido en grasas, una alta concentración de proteínas y contienen vitaminas. En este argumento los hongos silvestres comestibles podrían ser catalogados como especies de suma importancia en la conservación de algunas especies arbóreas que depende de ellos [12]. En síntesis, el conocimiento etnomicológico es cada vez mayor y está centrado principalmente entre las comunidades campesinas e indígenas del país [13].

Este trabajo tiene como objetivo proveer información acerca del uso de los hongos en cinco localidades pertenecientes a la Huasteca Hidalguense, y su modo de consumo por los pobladores.

\section{Material y Métodos}

La Huasteca Hidalguense se localiza al noreste del estado de Hidalgo, México, colinda con el estado de Veracruz. Presenta un clima caluroso y húmedo con lluvias en gran parte del año, ocasionando una vegetación verde y variada. Cuenta con un relieve tipo planicie con algunas montañas aisladas. Nunca excede los $800 \mathrm{msnm}$, pertenece a la planicie costera del Golfo de México. Las cinco localidades donde se realizaron los estudios pertenecen a tres Municipios, las cuales son: Ixtacuayo (Atlapexco); Mazahapa, San Juan (Huazalingo); Ahuehuetitla y Chalahuiyapa (Huejutla).
(Figura 1. Ubicación de los Municipios de Atlapexco, Huazalingo y Huejutla) [14].

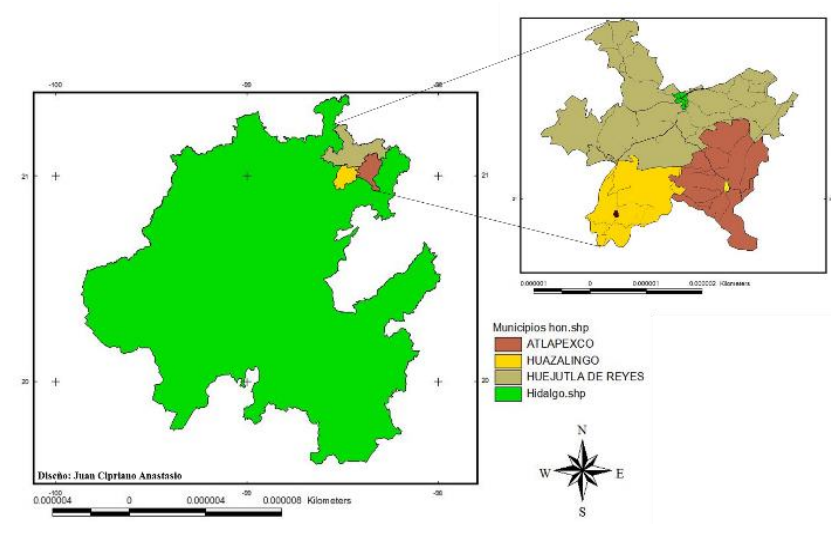

Figura 1. Ubicación de los Municipios de Atlapexco, Huazalingo y Huejutla.

Durante el periodo agosto-octubre del 2017, se realizaron visitas a las comunidades antes mencionadas. Con la ayuda de las autoridades de la comunidad se efectuaron un total de 52 entrevistas abiertas en total a personas mayores de 40 años, entrevistando a 32 mujeres y 20 hombres. Las encuestas realizadas se observan en el siguiente orden (Figura 2. Encuesta realizada a los pobladores).

\section{¿Conoce a los hongos? ¿Los ha consumido alguna vez? ¿Cómo los prepara? ¿Qué sabor tienen? ¿Cómo identifica los hongos entre los comestibles y los no comestibles? ¿En qué época podemos encontrar más hongos?}

\section{Figura 2. Encuesta realizada a los pobladores}

Se realizaron un total de seis colectas, dos en cada comunidad, con los pobladores en lugares de mayor humedad y diferentes tipos de sustratos en donde ellos señalaron que los extraen. Los ejemplares se colectaron siguiendo el protocolo recomendado por Lodge et al., [15] empleando una navaja para retirar los especímenes completos y se colocaron en bolsas de papel para ser transportados.

Una vez obtenidas las muestras, se utilizó la guía de determinación de hongos de Reyes-García et al., [16] y el manual de Micología básica de Frutis y Huidobro [17], para poder distinguir algunos rasgos característicos de estas especies tales como: color, tamaño, textura, olor y sabor. De esta manera se logró identificar a los hongos que son comestibles de esta zona y la manera de preparlos de acuerdo con la experiencia de los pobladores, y comprobando que los hongos encontrados son únicamente de autoconsumo. 


\section{Resultados}

Para las tres comunidades se colectaron un total de 12 ejemplares. Se obtuvieron nueve nombres comunes para los hongos, tres géneros y dos morfoespecies, las características utilizadas para identificarlos se presentan en la siguiente tabla (Tabla 1. Principales características de los géneros y especies de hongos.).

Tabla 1. Principales características de los géneros y especies de hongos.

\begin{tabular}{lllll}
\hline \multicolumn{5}{c}{ Caracteristicas } \\
\hline $\begin{array}{c}\text { Cantharellus } \\
\text { sp. }\end{array}$ & $\begin{array}{c}\text { Pleurotus } \\
s p .\end{array}$ & \multicolumn{1}{c}{$\begin{array}{c}\text { Auricularia } \\
\text { sp. }\end{array}$} & $\begin{array}{c}\text { Ustilago } \\
\text { maydis }\end{array}$ & $\begin{array}{l}\text { Agaricus } \\
\text { bisporus }\end{array}$ \\
\hline $\begin{array}{l}\text { Sombrero con } \\
\text { pliegues }\end{array}$ & $\begin{array}{l}\text { Laminas } \\
\text { decurrentes }\end{array}$ & $\begin{array}{l}\text { Fructificación } \\
\text { gelatinosa }\end{array}$ & $\begin{array}{l}\text { Membrana } \\
\text { lisa y fina }\end{array}$ & Anillo \\
\hline Esporas & Esporas & Esporas & Esporas & Laminas \\
amarilla-clara & blancas & blancas & globosas & carnosas \\
\hline Pie grueso y & Pie & Pie fijo & Adherido al & Pie \\
carnoso & lateral & al sustrato & maíz & fibroso \\
\hline Sin volva & Sin volva & carpóforo en & Color gris & Sin volva \\
& ni anillo & forma de copa & & \\
\hline
\end{tabular}

El que se les da a los hongos es alimenticio, presentándose siete maneras de preparado: tlapanile, guisado, en caldo chilahuile, en tamales hechos con hoja de papatla o plátano, quesadillas, adobo y hervidos. Los hongos más utilizados son los géneros Cantharellus y Pleurotus, comúnmente conocidos como hongo amarillo y blanco. Con la información recabada los hongos tienen un sabor similar al pescado. Cabe mencionar que el champiñón solo lo adquieren en los mercados. El modo de preparación tamal y guisado es el más utilizado, siendo el menos común en adobo y hervidos con sal (Tabla 2. Nombres comunes y modos de preparación de los hongos. Tlapanile $(\mathrm{T})$, guisado $(\mathrm{G})$, caldo $(\mathrm{C})$, tamales $(\mathrm{T})$, quesadillas (Q), adobo (A) y hervidos (H).).

Tabla 2. Nombres comunes y modos de preparación de los hongos. Tlapanile (T), guisado (G), caldo (C), tamales (T), quesadillas (Q), adobo (A) y hervidos

(H).

\begin{tabular}{llllllll}
\hline \multicolumn{1}{c}{$\begin{array}{c}\text { Nombre } \\
\text { común }\end{array}$} & T & G & C & T & Q & A & H \\
\hline $\begin{array}{l}\text { 1. Chiquinte 1 } \\
\text { (Hongo amarillo) }\end{array}$ & X & X & X & X & X & - & - \\
$\begin{array}{l}\text { 2. Chiquinte 2 } \\
\text { (Hongo blanco) }\end{array}$ & X & X & X & - & - & - & - \\
3. Alauachiquinte & X & X & X & X & X & - & - \\
4. Hongo del jonote & X & X & X & X & X & - & - \\
5. Xochinananaca & X & & & & X & - & - \\
6. Huitlacoche & - & X & - & - & - & - & - \\
7. Rebozuelo & - & - & - & - & - & X & - \\
8. Oreja de ratón & X & - & - & - & - & - & X \\
9. Champiñones & - & - & - & - & X & - & - \\
\hline
\end{tabular}

De acuerdo con la información recabada, algunos nombres comunes hacen referencia a la misma especie o género. En cuanto a su temporada, son colectados mayormente en época de lluvias y en diversos sustratos. Así mismo hacen mención que en la luna nueva se realiza la colecta de las fructificaciones. El árbol de chaca pertenece a la especie Bursera simaruba. El hongo conocido como oreja de ratón solo fue mencionado y no se encontró el fruto durante los recorridos (Tabla 3. Listado de especies y su época de recolección. Sustrato: bosque de encino (BE), árbol de chaca (AC), cortezas de los árboles y troncos (CT) y cultivo de maíz (CM). No aplica (NA).).

Tabla 3. Listado de especies y su época de recolección. Sustrato: bosque de encino (BE), árbol de chaca $(A C)$, cortezas de los árboles y troncos (CT) y cultivo de maíz (CM). No aplica (NA).

\begin{tabular}{lccc}
\hline \multicolumn{1}{c}{ Nombre común } & Especie & Temporada & Sustrato \\
\hline $\begin{array}{l}\text { 1. Chiquinte amarillo, } \\
\text { rebozuelo o }\end{array}$ & Cantharellus $s p$. & Lluvias & BE \\
$\begin{array}{l}\text { Xochinanacatl } \\
\text { 2. Chiquinte blanco ú }\end{array}$ & Pleurotus sp. & Lluvias y & AC \\
hongo de jonote & & luna nueva & \\
$\begin{array}{l}\text { 3. Oreja de cochino } \\
\text { 4. Huitlacoche }\end{array}$ & Auricularia sp. & Lluvias & CT \\
5. Oreja de ratón & Ustilago maydis & Lluvias & CM \\
& & Lluvias y & AC \\
& & luna nueva & \\
\hline
\end{tabular}

La diferenciación de los hongos comestibles de los no comestibles fue con base a los a los colores mencionados por los pobladores, comestibles: morado, blanco y amarillo, no comestibles: rojo y gris.

\section{Conclusiones}

Los pobladores de las cinco localidades aún conservan conocimiento sobre los hongos silvestres comestibles, obteniendo nueve nombres comunes para estos. Se determinaron taxonómicamente tres géneros y dos morfoespecies. Debido a la falta de material, no se realizó un análisis genético y por lo tanto solo se logró la identificación de los ejemplares colectados a nivel taxonómico de género y morfoespecie. en cuanto al huitlacoche su nombre científico es muy conocido, solo se desconoce la variedad que se consume en esta zona. En cuanto al hongo Champiñón solo se adquiere en los mercados. La época de mayor obtención es en la temporada de lluvias y luna nueva, finalmente la población identifica a los hongos comestibles y no comestibles a través de los colores y sabores, siendo el sabor similar al pescado. 


\section{Discusión}

En la presente investigación se obtuvieron tres géneros y dos morfoespecies: Cantharellus sp. (Hongo amarillo), Pleurotus sp. (Hongo blanco), Auricularia sp. (Hongo oreja de cochino), Ustilago maydis (Huitlacoche) y Agaricus bisporus (Champiñón). Estas dos últimas se tomaron como morfoespecie, por sus características morfológicas comunes.

Así mismo se obtuvieron siete formas de guisado [18]. Es importante mencionar que La población náhuatl que habita en la localidad de Chalahuiyapa, aún conserva el conocimiento sobre algunos hongos silvestres comestibles, el consumo de estas especies de hongos silvestres se realiza durante el verano y parte del otoño debido a la afluencia de las lluvias y dependiendo de la abundancia en la temporada es el consumo que se realiza [9]. Es importante mencionar que no existen trabajos etnomicológicos en la zona con los cuales podamos contrastar nuestros resultados, pues es un grupo que tiene poco interés en la Huasteca. Existen siete formas de preparar a los hongos, siendo la más común de cocinarlos fritos y en mole [11]. Mariaca-Méndez et al., [19] hace referencia que en otros lugares como en el sureste de México, el Huitlacoche se consume en caldo y tamales, variando el tipo de consumo en esta región que solo hacen referencia a guisado.

La población conoce a los hongos que son comestibles por su forma y tipo de sustrato en el que crecen, de acuerdo con Hernández-Velázquez [4] se identifican mucho mejor a los hongos comestibles, y no a los que son tóxicos, un dato importante es que el género Pleurotus, Cantharellus y Auricularia son uno de los más importantes para este Estado [10] y se consume mucho en esta región. Estas especies comparten el privilegio de ser las que la gente reconoce en mayor medida, prefiere y consume en mayor cantidad, debido, entre otras cosas, a sus características de abundancia y, sobre todo, su sabor [20]. Los hongos silvestres comestibles crecen sobre cinco tipos de sustrato: árbol de Chaca (Bursera simaruba), bosque de encinos (Quercus sp.), corteza de árboles y troncos, cafetales y planta de maíz, concordando con Ruan-Soto et al., [2] estos pueden variar dependiendo el tipo de vegetación y sustrato. En esta localidad los hongos son obtenidos por colecta directa, los autoconsumen y no los utilizan para la venta [21].

Lo anterior nos habla de lo importante y necesario que resulta realizar aproximaciones meticulosas en los procesos de alimentación, comercialización y revisión taxonómica de estos recursos alimenticios [3]. Los pobladores coinciden que antiguamente los hongos eran más abundantes y reemplazaban la carne en su dieta. La mayoría de ellos fueron alimentados por sus padres y/o abuelos con hongos silvestres comestibles, algunos de ellos alimentan a sus hijos de igual forma [22], a su vez, es importante mencionar que este aprovechamiento de las especies silvestres es simple y rudimentario [23].

\section{Referencias}

[1] Herrera, T. y M. Ulloa. 1990. El Reino de los Hongos, micología básica y aplicada. UNAM-Fondo de Cultura Económica, México, D. F. 552 p.

[2] Ruan-Soto, F., Garibay-Orijel, R. y Cifuentes, J. 2006. Process and dynamics of traditional selling wild edible mushrooms in tropical Mexico. Journal of Ethnobiology and Etnnomedicine 2(3).

[3] López-Ramírez, A. 1987. Hongos comestibles y medicinales de México. Ed. Posada. México. 21-33 pp.

[4] Hernández-Velázquez, H. 2007. Macromicetos de una región del Mineral el Chico, Hidalgo: Una aproximación a la Etnomicología. Tesis de Licenciatura en Biología, Universidad Autónoma del estado de Hidalgo, Pachuca 118-122 pp.

[5] Alexopoulus, C. J. 1996. Introductory micology, New York, Wiley. 108$123 \mathrm{pp}$.

[6] Durand, S. 1997. "Los hongos", Mundo Científico. Vol. 185, 1080-1083 pp.

[7] Mariaca-Méndez, R.; L. Del C., Silva-Pérez y C.A., Castaños-Montes. 2001. Proceso de recolección y comercialización de hongos comestibles silvestres en el Valle de Toluca, México. Ciencia Ergo Sum, vol. 8, número uno, Universidad Autónoma del Estado de México. Toluca, México, 30-40.

[8] Villareal, L. y J. Pérez-Moreno. 1989. Los hongos comestibles silvestres de México, un enfoque integral. Micología Neotropical Aplicada 2:77-114.

[9] Hernández-Rico, G. N. y Moreno-Fuentes, A. 2010. Hongos comestibles del género amanita en el mercado de Acaxochitlán, Hidalgo, México. Etnobiología 8: 31-38.

[10] Moreno-Fuentes, A. y E. Bautista-Nava. 2006. El "hongo blanco patón", Pleurotus albidus, en Hidalgo. Su primer registró en México. Rev. Mex. Mic. 22: 41-47.

[11] Bautista-Nava, E.; A., Moreno-Fuentes; Ma.T., Pulido-Silva; R., Valadez-Azúa y R., Ávila-Pozos. 2010. Bases bioculturales para el aprovechamiento y conservación de los hongos silvestres comestibles en el municipio de Tenango de Doria, Hidalgo, México. En: Sistemas Biocognitivos Tradicionales Paradigmas en la conservación Biológica y el Fortalecimiento Cultural. Moreno-Fuentes, A.; Ma.T., Pulido-Silva; R., Mariaca- Méndez; R., Valadez-Azúa; P., Mejía Correa y T.V., Gutiérrez-Santillán (eds). México. 226-231.

[12] Isasi, E. 2011. Los conceptos de especies indicadoras, paraguas, banderas y claves: su uso y abuso en ecología de la conservación. Interciencia 36(1): 31-38.

[13] Henao, L. y A. Ruiz. 2006. Investigación y Gestión local de robledales alrededor del uso tradicional de macromicetos en la cordillera Oriental colombiana. En: Solano, C. y N. Vargas (ed.). Memorias del I Simposio de Robles y Ecosistemas Asociados. Bogotá. Fundación Natura y Pontificia Universidad Javeriana. 
[14] INEGI. 2015. Anuario estadístico del estado de Hidalgo. Instituto Nacional de Estadística y Geografía, México. 606 p.

[15] Lodge DJ, Ammirati JF, O'Dell TE, Mueller GM. 2004. Collecting and describing macrofungi. In: Mueller GM, Bills GF, Foster MS. Biodiversity of fungi: inventory and monitoring methods. Elsevier Academic Press, Oxford, UK. 777 p.

[16] Reyes-García, M. G., Gómez-Peralta, M. y Zamora-Equihua, V. 2009. Guía de Hongos de los Alrededores de Morelia. Museo de Historia Natural "Manuel Martínez Solórzano". Universidad Michoacana de San Nicolás de Hidalgo. 377 p.

[17] Frutis, M. I. y Huidobro, S. M. E. 2013. Micología Básica, Manual Teórico Práctico. Ed. FES Iztacala, Segunda edición. Universidad Nacional Autónoma de México. Facultad de Estudios Superiores Iztacala. 203 p.

[18] Cipriano-Anastasio, J. Galván, G. R., López, M. A., Hernández, A. G. Marcos, M. R., Martínez, H. E. 2018. Uso de los hongos en cinco Localidades de la Huasteca Hidalguense. XI Congreso Mexicano de Etnobiología. Morelia, Michoacán (Memoria de congreso).

[19] Mariaca-Méndez, R.; Ruan-Soto, F. y Cano-Contreras, E. J. 2008 Conocimiento tradicional de Ustilago maydis en cuatro grupos mayenses del sureste de México. Etnobiología 6 9-23

[20] Ruan-Soto, F., Hernández-Maza, M. y Pérez-Ovando, E. 2013. Estado actual del conocimiento de la diversidad fúngica en Chiapas, en Comisión Nacional para el Conocimiento y Uso de la Biodiversidad, La biodiversidad en Chiapas: Estudio de estado, México, conabio/Gobierno del Estado de Chiapas, pp. 75-83.

[21] Tovar, J. A. y Valenzuela, R. 2006. Los Hongos del Parque Nacional Desierto de los Leones. Gobierno del Distrito Federal/Secretaría del Medio Ambiente/Parque Nacional Desierto de los Leones. México, D.F, México. Pp. 37-82.

[22] Ruan-Soto, F., J. Cifuentes, R. Mariaca, F. Limón y S. Pérez-Ramírez. 2009. Uso y manejo de hongos silvestres en dos comunidades de la Selva Lacandona, Chiapas, México. Revista Mexicana de Micología 29: 61-72.

[23] Garibay-Orijel, Roberto, Felipe Ruan-Soto y Emma Estrada-Martínez. 2010. El conocimiento micológico tradicional, motor para el desarrollo del aprovechamiento de los hongos comestibles y medicinales. Red Latinoamericana de Hongos Comestibles y medicinales/ colpos/ unsconacyt/ amc/ uaem/ upaep/ iminap, Puebla, pp. 243-270 pp. 